\title{
Analysis of 3-phase Induction Motor considering Current Regulator using DQ Transformation with Matrix Vector
}

\author{
Sun-Ki Hong* and Yoo-Chung Na**
}

\begin{abstract}
Induction machines are being used in industry and $d q$ transformation from 3 phase of $a, b, c$ is commonly used to analyze these machines. The equivalent circuits of $d$ and $q$ axis are however generally cross coupled and not simple to analyze. In this study, an analysis method of 3 ph induction motor considering current regulator using $d q$ transformation and matrix vector is proposed and it can explain the 3 ph induction motor physically. This model does not need the separating process of $d$ and $q$ components. With this technique, the model becomes simple, is easy to understand in physical, and can get the same results with those from the other $d q$ models. These simulation results of the proposed model are compared with those of other models for the conformation of the proposed method.
\end{abstract}

Keywords: DQ transformation, Matrix vector, 3ph Induction motor, Current regulator

\section{Introduction}

3 ph induction machines are very widely being used in industrial area. DQ transformation is commonly used to analyze the induction machines' characteristics [1]-[4]. The equivalent circuits of $d$ and $q$ axis are however generally cross coupled and that makes it difficult to calculate the characteristics of the machine. In this study, an analysis method of 3 ph induction motor including current regulator using $d q$ transformation with matrix vector is proposed. The model parameters can be matched with the 3 ph induction machine physically and it does not need the separating process of $d$ and $q$ components. With this, the model becomes simple and is easy to understand in physical. These simulation results of the proposed model are compared with those of other models for the conformation of the proposed method.

\section{Conventional Induction Motor Modeling}

2.13 ph induction motor model using $\psi=\omega_{b} \lambda$ at arbitrary reference frame

The $d q$ voltage equations of stator and rotor from 3 phases become (1), (2) [3]. (3), (4) shows the magnetic flux linkage and torque equations.

\footnotetext{
* Dept. of Digital Control Engineering, Hoseo University, Korea. (skhong@hoseo.edu)

** Dept. of Digital Control Engineering, Hoseo University, Korea. (@ naver.com)
}

Received 16 April 2014 ; Accepted 24 April 2014

$$
\begin{aligned}
& v_{q s}=P \lambda_{q s}+\omega \lambda_{d s}+r_{s} i_{q s} \\
& v_{d s}=p \lambda_{d s}+\frac{\omega}{\omega_{b}} \lambda_{q s}+r_{s} i_{d s} \\
& v_{q r}^{\prime}=p \lambda_{q r}^{\prime}+\left(\omega-\omega_{r}\right) \lambda_{d s}^{\prime}+r_{r}^{\prime} i_{q r}^{\prime} \\
& v_{d r}^{\prime}=p \lambda_{d r}^{\prime}+\left(\omega-\omega_{r}\right) \lambda_{q s}^{\prime}+r_{r}^{\prime} i_{d r}^{\prime} \\
& {\left[\begin{array}{l}
\lambda_{q s} \\
\lambda_{d s} \\
\lambda_{q r}^{\prime} \\
\lambda_{d r}^{\prime}
\end{array}\right]=\left[\begin{array}{cccc}
L_{l s}+L_{m} & 0 & L_{m} & 0 \\
0 & L_{l s}+L_{m} & 0 & L_{m} \\
L_{m} & 0 & L_{l s}+L_{m} & 0 \\
0 & L_{m} & 0 & L_{l s}+L_{m}
\end{array}\right]\left[\begin{array}{c}
i_{q s} \\
i_{d s} \\
i_{q r}^{\prime} \\
i_{d r}^{\prime}
\end{array}\right]} \\
& T_{e m}=\frac{3}{2} \frac{P}{2}\left(\lambda_{d s} i_{d s}-\lambda_{q s} i_{d s}\right) \\
& =\frac{3}{2} \frac{P}{2} L_{m}\left(i_{d r}^{\prime} i_{q s}-i_{q r}^{\prime} i_{d s}\right)
\end{aligned}
$$

Where, $p$ is differential operator, $P$ is number of poles, $\omega$ is the speed of frame and $\omega_{r}$ is the speed of rotor, $\lambda$ is flux linkage, $L_{l s}, L_{l r}$ are leakage inductance, $T_{e m}$ is developed motor torque and " means the transferred value from rotor to stator side. From these equations, Ong [3] adopted flux linkage per sec $\psi=\omega_{b} \lambda$ which dimension is voltage. With this, (1) (4) become (5) (8) [4] and the flux linkage equation (3) is changed to flux linkage per second equation (7)..

$$
\begin{aligned}
& v_{q s}=\frac{p}{\omega_{b}} \psi_{q s}+\frac{\omega}{\omega_{b}} \psi_{d s}+r_{s} i_{q s} \\
& v_{d s}=\frac{P}{\omega_{b}} \psi_{d s}+\frac{\omega}{\omega_{b}} \psi_{q s}+r_{s} i_{d s}
\end{aligned}
$$




$$
\begin{gathered}
v_{q r}^{\prime}=\frac{p}{\omega_{b}} \psi_{q r}^{\prime}+\left(\frac{\omega-\omega_{r}}{\omega_{b}}\right) \psi_{d s}^{\prime}+r_{r}^{\prime} i_{q r}^{\prime} \\
v_{d r}^{\prime}=\frac{p}{\omega_{b}} \psi_{d r}^{\prime}+\left(\frac{\omega-\omega_{r}}{\omega_{b}}\right) \psi_{q s}^{\prime}+r_{r}^{\prime} i_{d r}^{\prime} \\
{\left[\begin{array}{c}
\psi_{q s} \\
\psi_{d s} \\
\psi_{q r}^{\prime} \\
\psi_{d r}^{\prime}
\end{array}\right]=\left[\begin{array}{cccc}
x_{l s}+x_{m} & 0 & x_{m} & 0 \\
0 & x_{l s}+x_{m} & 0 & x_{m} \\
x_{m} & 0 & x_{l s}+x_{m} & 0 \\
0 & x_{m} & 0 & x_{l s}+x_{m}
\end{array}\right]\left[\begin{array}{l}
i_{q s} \\
i_{d s} \\
i_{q r}^{\prime} \\
i_{d r}^{\prime}
\end{array}\right] \text { (7) }} \\
T_{e m}=\frac{3}{2} \frac{P}{2 \omega_{b}}\left(\psi_{d s} i_{q s}-\psi_{q s} i_{d s}\right)
\end{gathered}
$$

\subsection{3ph Induction motor model using flux linkage $\lambda$}

As mentioned in 2.1, $\psi$ model needs somewhat complex mathematical equations and has physical ambiguities in the model block. In this section, flux linkage $\lambda$ itself is not modified but used directly to derive the integration form equations as (9) - (11) and (3) for physical meaning.

$$
\begin{gathered}
i_{q s}=\frac{1}{L_{l s}+L_{m}} \int\left(v_{q s}-r_{s} i_{q s}-\omega \lambda_{d s}-L_{m} \frac{d}{d t} i_{q r}^{\prime}\right) d t \\
i_{d s}=\frac{1}{L_{l s}+L_{m}} \int\left(v_{d s}-r_{s} i_{d s}-\omega \lambda_{q s}-L_{m} \frac{d}{d t} i_{d r}^{\prime}\right) d t \\
i_{q r}^{\prime}=\frac{1}{L_{l r}^{\prime}+L_{m}} \int\left(-r_{r}^{\prime} i_{q r}^{\prime}-\left(\omega-\omega_{r}\right) \lambda_{d r}^{\prime}-L_{m} \frac{d}{d t} i_{q s}\right) d t \\
i_{d r}^{\prime}=\frac{1}{L_{l r}^{\prime}+L_{m}} \int\left(-r_{r}^{\prime} i_{d r}^{\prime}-\left(\omega-\omega_{r}\right) \lambda_{q r}^{\prime}-L_{m} \frac{d}{d t} i_{d s}\right) d t \\
T_{e m}=\frac{3}{2} \frac{P}{2} L_{m}\left(i_{q s}^{s} i_{d r}^{s}-i_{d s}^{s} i_{q r}^{s}\right)
\end{gathered}
$$

This model is derived using $\lambda$ which has physical meaning although it looks a little bit more complex. Actually it doesn't have sub-blocks, so it becomes simpler than the previous model using $\psi=\omega_{b} \lambda$.

\subsection{3ph induction motor model using complex vector}

Complex vector has real and imaginary axis. In $3 \mathrm{ph}$ induction motor modeling, $q$-axis is real and $d$-axis is imaginary axis. This kind of modeling becomes simpler than the common $d q$ transformation method because it does not need to separate the $d$ and $q$ components With Simulink tool, it is easy to realize to complex vector model and the complex calculation is possible in the model block. For example, the $d q$ voltage equation can be expressed as $\boldsymbol{v}_{q d}=v_{q}+j v_{d}$, where the bold character means complex vector. The following equations of (12) and (13) show the voltage and flux linkage equations of complex vector form in stator and rotor [5].

$$
\begin{aligned}
\boldsymbol{v}_{q d} & =r_{s} \boldsymbol{i}_{q d}+\frac{d}{d t} \boldsymbol{\lambda}_{q d}+j \omega \boldsymbol{\lambda}_{q d} \\
\boldsymbol{v}_{q d r} & =r_{\mathrm{s}} \boldsymbol{i}_{d s}+\frac{d}{d t} \boldsymbol{\lambda}_{q d}+j \omega \boldsymbol{\lambda}_{q d} \\
\boldsymbol{\lambda}_{q d} & =L_{s} \boldsymbol{i}_{q d}+L_{m} \boldsymbol{i}_{q d r} \\
\boldsymbol{\lambda}_{q d r} & =L_{r} \boldsymbol{i}_{q d r}+L_{m} \boldsymbol{i}_{q d}
\end{aligned}
$$

In this approach, current complex vector $\boldsymbol{i}_{q d}$ and magnetic flux linkage complex vector $\lambda_{q d r}$ become the variables to solve therefore $\boldsymbol{i}_{q d r}$ and $\boldsymbol{\lambda}_{q d}$ need to be erased. With (13), the following equation of (14) is derived.

$$
\begin{gathered}
\lambda_{q d}=\sigma L_{s} \boldsymbol{i}_{q d}+\frac{L_{m}}{L_{r}} \lambda_{q d r} \\
\sigma=1-L_{m}^{2} / L_{s} L_{r}
\end{gathered}
$$

$\sigma$ is defined as (15) for simplicity. (14) is substituted for (12) and the following differential equations are derived.

$$
\begin{aligned}
\sigma \frac{d}{d t} \boldsymbol{i}_{q d} & =\boldsymbol{v}_{q d}-\left(r_{s}+j \omega \sigma L_{s}\right) \boldsymbol{i}_{q d} \\
& -\frac{L_{m}}{L_{r}}\left(\frac{d}{d t} \boldsymbol{\lambda}_{q d r}+j \omega \boldsymbol{\lambda}_{q d r}\right) \\
\frac{d}{d t} \boldsymbol{\lambda}_{q d r} & =-\frac{r_{r}}{L_{r}}-j\left(\omega-\omega_{r}\right) \boldsymbol{\lambda}_{q d r}+r_{r} \frac{L_{m}}{L_{r}} \boldsymbol{i}_{q d}
\end{aligned}
$$

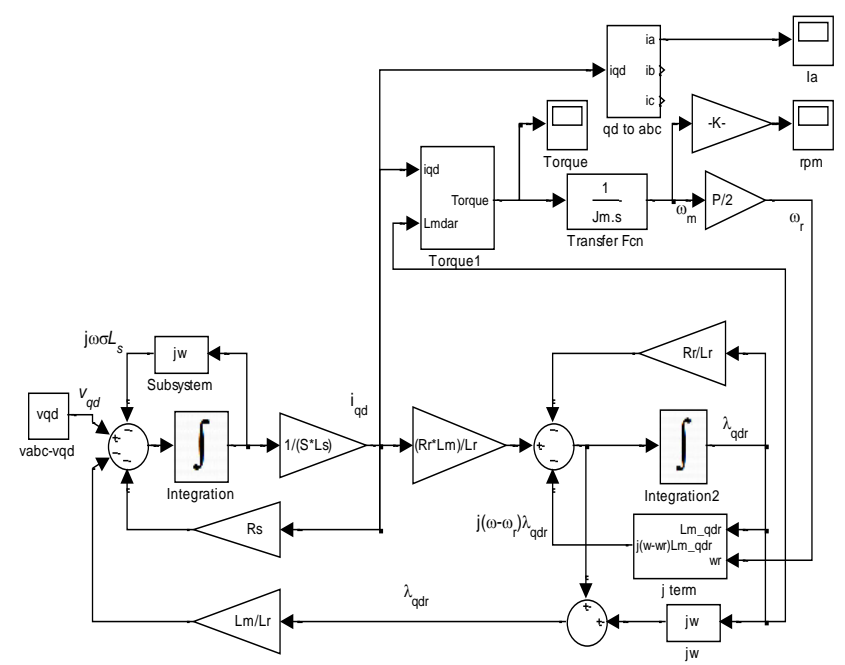

Fig. 1. IM model using complex vector for arbitrary ref. frame.

Fig. 1 shows the 3 ph induction motor model derived from (16) and (17). This model does not need to extra parameter like $\psi$ and does not look complex as the flux linkage model [3]. Each icon has physical meaning and is 
easily realized as the model in Fig. 1. In the figure, the integral symbol is a sub block that has two integral processes of each $q$ and $d$ axis equation. After the integrations, they are combined again to make complex vector.

With these results, the IM modeling with complex vector and $d q$ transformation has simple structure, is easy to debug and to analyze IM characteristics.

\subsection{3ph induction motor model using matrix vector}

The complex vector model of IM is very useful to analyze induction machine, however the model is not able to apply to permanent magnet synchronous machine because the applied machine with complex vector method needs to be symmetric.

Eq. (17)-(20) shows the 3 ph induction motor's stator, rotor, and field equations where bold character means not complex but matrix form and (3) is the flux linkage equation.

$$
\begin{aligned}
\boldsymbol{V}_{q d} & =\boldsymbol{r}_{s} \boldsymbol{i}_{q d}+\frac{d}{d t} \boldsymbol{\lambda}_{q d}+\boldsymbol{j} \omega \boldsymbol{\lambda}_{q d} \\
0 & =\boldsymbol{r}_{r} \boldsymbol{i}_{q d r}+\frac{d}{d t} \boldsymbol{\lambda}_{q d r}+\boldsymbol{j}\left(\omega-\omega_{r}\right) \boldsymbol{\lambda}_{q d r} \\
\boldsymbol{V}_{f r} & =\boldsymbol{r}_{f} \boldsymbol{i}_{f r}+\frac{d}{d t} \boldsymbol{\lambda}_{q d r} \\
\lambda_{q d r} & =\boldsymbol{L}_{r} \boldsymbol{i}_{q d r}+\boldsymbol{L}_{m}\left(\boldsymbol{i}_{q d}+\boldsymbol{i}_{f r}\right)+\boldsymbol{\lambda}_{p m}
\end{aligned}
$$

In those equations, bold $\boldsymbol{j}$ is not just a imaginary symbol but is matrix vector form as (21). $\boldsymbol{L}_{s}$ is also a matrix as follows.

$$
\begin{aligned}
\boldsymbol{j} & =\left[\begin{array}{cc}
0 & 1 \\
-1 & 0
\end{array}\right] \\
\boldsymbol{L}_{s} & =\left[\begin{array}{ll}
L_{q q} & L_{q d} \\
L_{d q} & L_{d d}
\end{array}\right]
\end{aligned}
$$

With these equations, $3 \mathrm{ph}$ induction motor model for arbitrary reference frame with matrix vector can be induced. (23) and (24) shows the differential equations from (17)(20). This model can also be applied to PM synchronous motor because it considers the field current PM flux linkage.

$$
\begin{aligned}
& \frac{\mathrm{d}}{\mathrm{dt}} \boldsymbol{L}_{s} \boldsymbol{\sigma} \boldsymbol{i}_{q d}=\boldsymbol{V}_{q d}-\boldsymbol{r}_{s} \boldsymbol{i}_{q d}-\boldsymbol{j} \omega \boldsymbol{L}_{s} \boldsymbol{\sigma} \boldsymbol{i}_{q d} \\
& -\left(\frac{\mathrm{d}}{\mathrm{dt}} \boldsymbol{L}_{m} \boldsymbol{L}_{r}^{-1} \lambda_{q d r}+\boldsymbol{j} \omega \boldsymbol{L}_{m} \boldsymbol{L}_{r}^{-1} \boldsymbol{\lambda}_{q d r}\right)-\left(\boldsymbol{I}-\boldsymbol{L}_{m} \boldsymbol{L}_{r}^{-1}\right)
\end{aligned}
$$

$$
\begin{gathered}
\left\{\frac{d}{d t}\left(\boldsymbol{L}_{m} \boldsymbol{i}_{f r}+\boldsymbol{\lambda}_{p m}\right)+\boldsymbol{j} \omega\left(\boldsymbol{L}_{m} \boldsymbol{i}_{f r}+\lambda_{p m}\right)\right\} \\
\frac{d}{d t} \boldsymbol{\lambda}_{q d r}=\boldsymbol{r}_{r} \boldsymbol{L}_{r}^{-1}\left[\boldsymbol{L}_{m} \boldsymbol{i}_{q d}+\left(\boldsymbol{L}_{m} \boldsymbol{i}_{f r}+\boldsymbol{\lambda}_{p m}\right)\right. \\
\left.-\left\{\boldsymbol{I}+\boldsymbol{L}_{r} \boldsymbol{r}_{r}^{-1} \boldsymbol{j}\left(\omega-\omega_{r}\right)\right\} \boldsymbol{\lambda}_{q d r}\right]
\end{gathered}
$$

Fig. 2 shows the AC machine model made from (23)(24). It doesn't have cross coupling and has simple structure and is physically well explained.

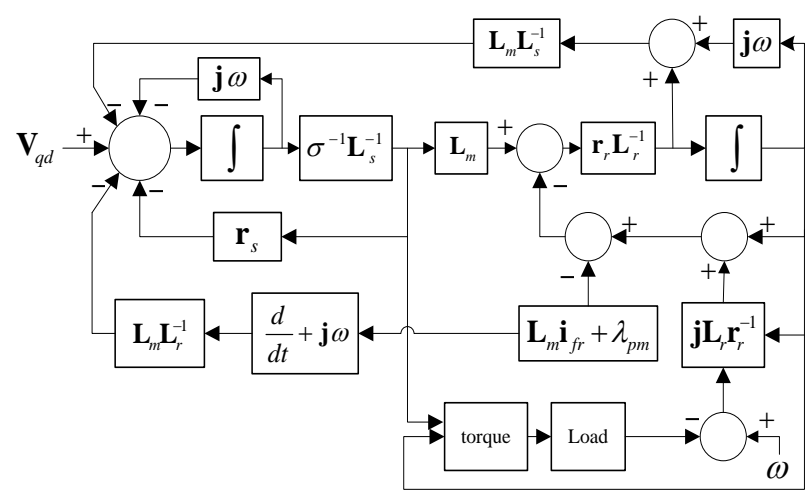

Fig. 2. Generalized AC machine model.

\section{Current Regulator Model with Matrix Vector}

Due to the previous simple 3 ph induction motor model, the simulation of motor characteristics becomes easy and the machine model can be combined with current controller without any modification. Figure 3 shows the 3 ph induction motor model with current controller [3].

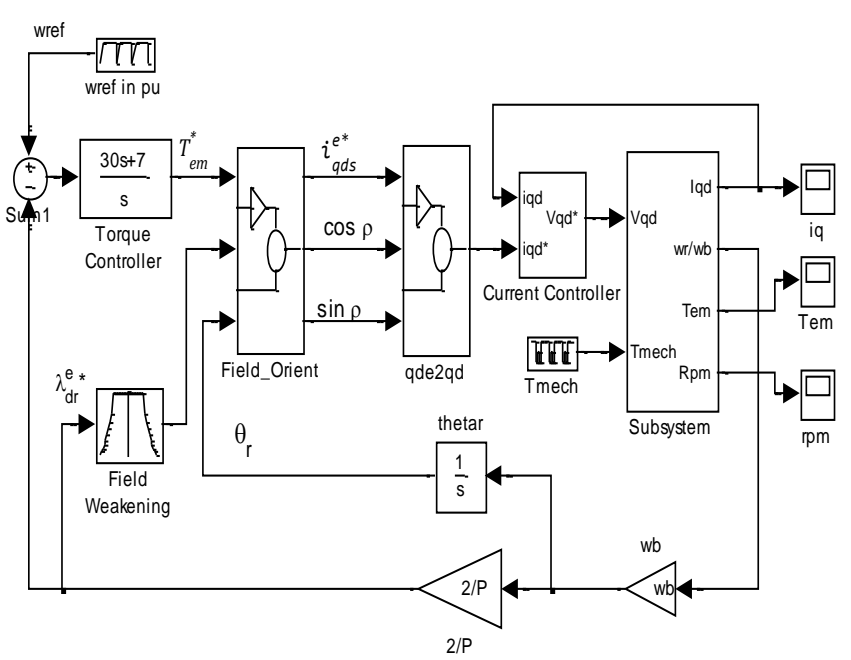

Fig. 3. Generalized AC machine model with current controller.

The current controller is the proportional-gain controller which is generally used in current control. At first, speed command wref is applied and this is compared with the 
motor speed to generated torque command $T_{e m}^{*}$. In the field oriented block, $q$ and $d$ axis currents for synchronous ref. frame are transformed to the stationary ref. frame currents $\boldsymbol{i}_{q d s}^{*}$, which can be seen in Fig. 4 .

This current command vector is compared with the feedback current vector of $i_{q d s}$ to generate the voltage command vector $v_{q d}^{*}$. As mentioned before, the current controller adopts the proportional-gain control. The last block is same with Fig. 2.

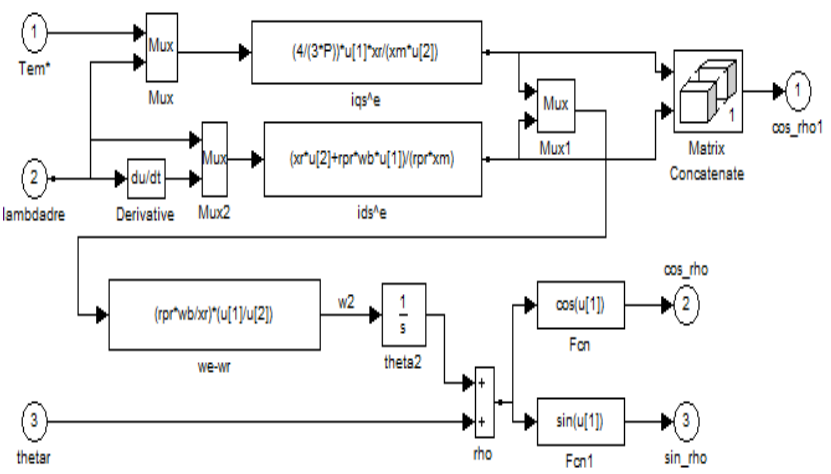

Fig. 4. Field orient block in Fig. 3.

\section{Simulation and Comparison}

The simulation is executed for a 3 ph induction motor to compare the results with each other. The specification of sample IM is that $220 \mathrm{~V}, 60 \mathrm{~Hz}, 4$ poles, stator and rotor resistance are $0.435 \Omega$ and $0.816 \Omega$, leakage and magnetization inductance are $2 \mathrm{mH}$ and $69.3 \mathrm{mH}$ and moment of inertia is 0.089 . Fig. 5 shows the simulation block when 3-phase voltage is applied to the induction motor of Fig. 2. The 3-phase voltage is transformed to $q d$ voltage and the $q d$ voltage is applied to the IM. The motor block in Fig. 2 becomes 3 ph induction motor when the permanent flux linkage is 0 and field current is 0 .

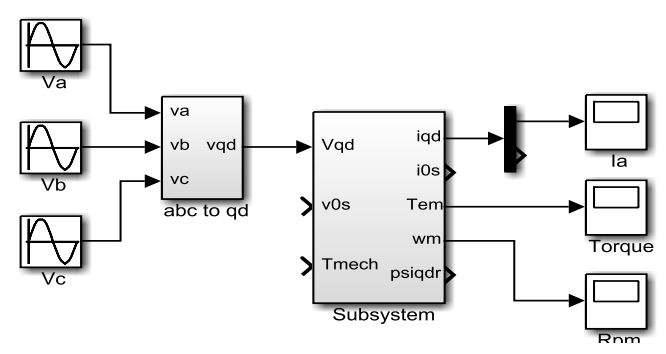

Fig. 5. Induction motor simulation block for 3-phase voltage.

Fig. 6(a) shows the speed response when the speed command is $1,800 \mathrm{rpm}$. Fig 6(b) shows the torque response waveform of an induction motor and the results are well matched with the results of other models [3, 4] because the sample specification is same.

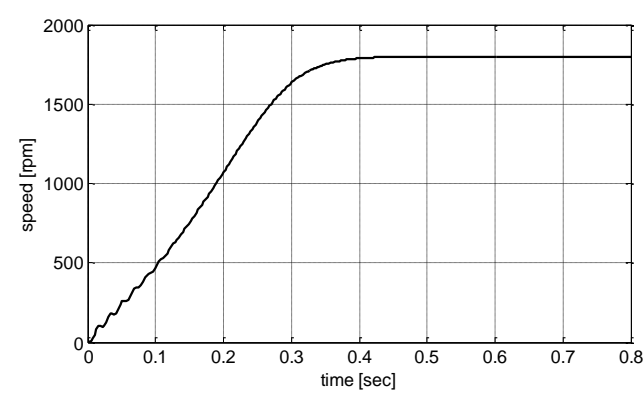

(a) speed response

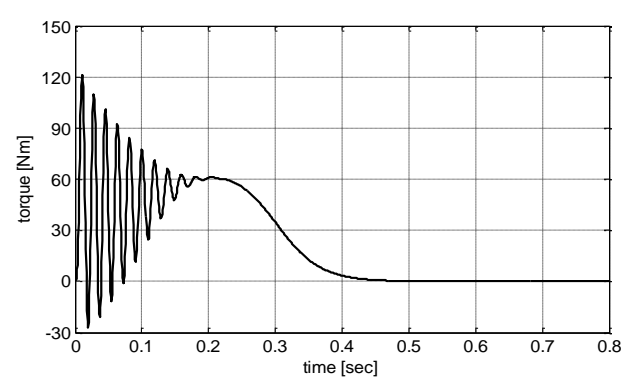

(b) torque response

Fig. 6. IM waveforms using matrix vector for stationary ref. frame.

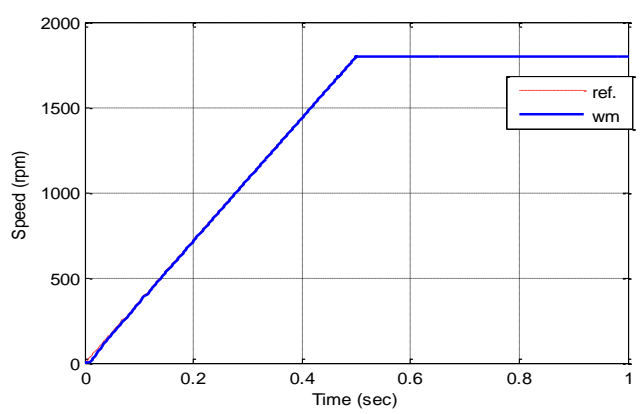

(a) speed response

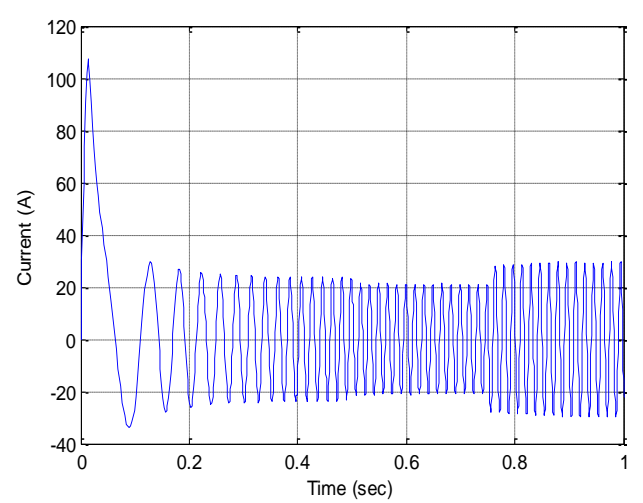

(b) current waveform of phase $a$

Fig. 7. IM waveforms under speed control with current controller. 
The block diagram shown in Fig. 2 has simple structure and can be applied to not only 3 ph induction motor but also permanent magnet type synchronous motor.

Fig. 7 shows the simulation results for the system of Fig. 3. Fig. 7(a) shows the comparison between speed reference and the response. The speed command is ramp input from 0 to $0.5(\mathrm{sec})$ and after that the speed command is constant as 1800 (rpm). They are well matched almost without overshoot because the input command is not step but ramp. Fig. 7(b) shows the $a$-phase current wave form. At 0.75 (sec), the load torque is applied and the phase current is increased to match with the load variance.

\section{Conclusions}

Analyzing method for 3 ph induction motor including current regulator using $d q$ transformation and matrix vector are surveyed. That 3 ph induction model is combined with current controller model and simulated for speed control. The proposed method looks very useful for 3 ph induction motor analysis and simulation because of its simple structure and even no problem to apply to asymmetric machine of SM.

\section{Acknowledgements}

This work was supported by the National Research Foundation of Korea(NRF) grant funded by the Korea government(MEST) (No.2010-0023043)

\section{References}

[1] H. Kim and R. D. Lorenz, "Synchronous frame PI current regulators in a virtually translated system," presented at 2004 IEEE IAS Annual Meeting, Seattle, WA, USA, 2004.

[2] D. W. Novotny and T. A. Lipo, Vector Control and Dynamics of AC Drives : Clarendon Press Oxford 1996.

[3] Chee-Mun Ong, "Dynamic Simulation of Electric Machinery”, PrenticeHall, 1997.

[4] S. Hong, "A Study on AC Machine Modeling using Complex Vector and d-q Transformation", KIEE Transactions on, vol. 61, No.11, pp.1601-1605, 2013.

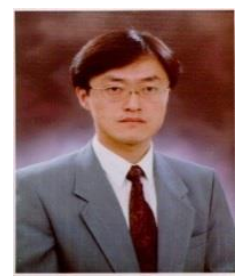

Sun-Ki Hong received his M.S. and the Ph.D in electrical engineering from Seoul National University in 1989, 1993. His special interests is the modeling and computation of hysteresis, design and analysis, control of electric machines.

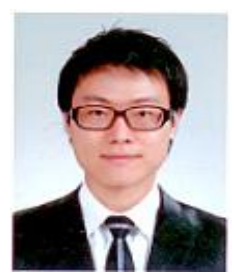

Yoo-Chung Na received B.S degree in system engineering from hoseo University. His research interests are analysis and modeling of electric machines, hardware programming, embedded controller, control of servo machine. 